\title{
ENTIRE FUNCTIONS OF EXPONENTIAL TYPE
}

\author{
R. P. BOAS, JR.
}

1. Introduction. An entire function $f(z)$ is said to be of exponential type if it is of order one and mean type; that is, if for some non-negative $c$ and every positive $\epsilon$ there is a number $A(\epsilon)$ such that

$$
|f(z)|<A(\epsilon) e^{(c+\epsilon)|z|}
$$

for all $z$. The smallest $c$ which can be used in (1) is called the type of $f(z)$. An alternative definition ${ }^{1}$ is

$$
c=\limsup _{n \rightarrow \infty}\left|f^{(n)}(z)\right|^{1 / n}
$$

it is immaterial which value of $z$ is used in (2). If (1) holds in a region of the $z$-plane, for example in an angle, $f(z)$ is said to be of exponential type $c$ in that region.

Functions of exponential type have been extensively studied, both for their own sake and for their applications. I shall discuss here a selection of their properties, chosen to illustrate how the restriction (1) on the growth of a function restricts its behavior in other ways. ${ }^{2}$

2. Representations. Various formulas are available for representing functions of exponential type. Some of these representations are useful for deriving results of the kind discussed later in this report; and they are of considerable interest for their own sake.

If $f(z)$ is an entire function satisfying (1), there is a function $\phi(w)$, analytic in $|w|>c$, such that

$$
f(z)=\int_{C} e^{z w} \phi(w) d w
$$

where $C$ is any contour containing $|w|=c$ in its interior. ${ }^{3}$ The function $\phi(w)$ is defined by either of the equivalent formulas

and

$$
\phi(w)=\frac{1}{2 \pi i} \sum_{n=0}^{\infty} \frac{n ! a_{n}}{w^{n+1}} \text { if } f(z)=\sum_{n=0}^{\infty} a_{n} z^{n}
$$

An address delivered before the New York meeting of the Society on April 4, 1942, by invitation of the Program Committee; received by the editors July 28, 1942.

${ }^{1}$ See $[10$, p. 241$]$. [10].

${ }^{2}$ For a report on functions of exponential type from another point of view, see

${ }^{3}$ See $[25$, pp. 578-586]. 


$$
\phi(w)=\frac{1}{2 \pi i} \int_{0}^{\infty} f(x) e^{-x w} d x
$$

$\Re(w)>c$.

More precisely, $C$ in (3) can be any curve surrounding the set of singular points of $\phi(w)$; the configuration of this set depends on the growth of $f(z)$ in various directions. In this report, however, I shall usually neglect the more refined results in which the shape of the set of singular points of $\phi(w)$ is taken into consideration. Any result involving the type of $f(z)$ will have refinements involving the growth of $f(z)$ in two or more directions.

However, the case in which $\phi(w)$ has singularities only along a line segment is of particular interest. If $f(z)$ belongs to $L^{2}$ on a line (for definiteness, the real axis), then

$$
f(z)=\int_{-c}^{c} e^{i z t} F(t) d t, \quad F(t) \in L^{2}(-c, c) ;
$$

and conversely. ${ }^{4}$ This result can be extended to other $L$-classes, though with some loss of elegance. If $f(z) \in L^{p}(-\infty, \infty)(1<p<2)$, then $f(z)$ has the representation (4) with $F(t) \in L^{p /(p-1)}$, but the converse is false. ${ }^{5}$ A necessary and sufficient condition $[23$, p. 111] for $f(z)$ to belong to $L^{p}(p>1)$ is that

$$
f(z)=z \int_{-c}^{c}[F(c)-F(t)] e^{i z t} d t-2 F(c) z^{-1} \sin c z
$$

where $F(t)$ is a continuous function of period $2 c$ whose Fourier coefficients $c_{n}$ satisfy

$$
\sum_{-\infty}^{\infty}\left|n c_{n}\right|^{p}<\infty .
$$

When $p=1$, we have the necessary and sufficient condition $[1, p$. 283]

$$
f(z)=\int_{-c}^{c} G(t) e^{i z t} d t
$$

where $G(t)$ has an absolutely convergent Fourier series and $G(c-)$ $=G(-c+)=0$.

For functions $f(z)$ bounded on the real axis, we have the representations (necessary but not sufficient) ${ }^{6}$

\footnotetext{
${ }^{4}$ [21, p. 13]. A sharper result is given by Plancherel and Pólya [23, p. 228].

$5[1$, p. 280]. The result is implicit in [23, p. 243].

$6\left[3\right.$, p. 151]. To obtain (5), apply (4) to $z^{-1}[f(z)-f(0)]$.
} 


$$
f(z)=f(0)+z \int_{-c}^{c} e^{i z t} F(t) d t, \quad F(t) \in L^{2}
$$

and also [2]

$$
f(z)=\int_{-c}^{c} e^{i z t} \frac{d^{2} F(t)}{d t} ;
$$

the notation in (6) is purely formal; what is meant is that the 2-transform $F(t)$ (in the sense of Bochner [9]) of $f(z)$ is necessarily linear outside $(-c, c)$. Both (5) and (6) have been applied in the study of functions of exponential type $[19,3]$.

For a function of exponential type in an angle, Macintyre [20] has given a useful representation by a contour integral.

3. Operators. A large number of the results mentioned in this report have to do with relations between the behavior of an entire function $f(z)$ and that of the functions obtained by operating on $f(z)$ with a sequence of operators $L_{n}$. In many cases, the $L_{n}$ are the powers $L^{n}$ of a single operator. The most interesting-certainly the most easily dealt with-operators $L$ appear to be those which are distributive and (in a suitable sense) continuous, which transform an entire function of exponential type less than $c$ into another function of type less than $c$, and which are permutable with differentiation. Associated with any such operator $L$ there is a function $\lambda(w)$, analytic in $|w|<c$, such that if $f(z)$ has the representation (3), then ${ }^{7}$

$$
L[f(z)]=\int_{C} e^{z w} \phi(w) \lambda(w) d w
$$

correspondingly, $L$ may be regarded as the differential operator of infinite order, $\lambda(d / d z)$. Examples which have been frequently discussed are $L[f(z)]=f(z+1), L[f(z)]=f^{\prime}(z), L[f(z)]=f(z+1)-f(z)$. The corresponding functions $\lambda(w)$ are $e^{w}, w$, and $e^{w}-1$.

Corresponding to any sequence of operators $L_{n}$ of this character, there is a uniqueness problem: given a sequence of complex numbers $\left\{a_{n}\right\}_{n=0}^{\infty}$, for what class of functions of exponential type does $L_{n}\left[f\left(a_{n}\right)\right]=0(n=0,1,2, \cdots)$ imply $f(z) \equiv 0$ ? A somewhat more general problem asks, given a set of circles $C_{n}$, whether at least one of the functions $L_{n}[f(z)$ ] is necessarily univalent in the corresponding $C_{n}{ }^{8}$

Because of the representation (7) for the operators $L_{n}$, uniqueness

\footnotetext{
${ }^{7}$ Boas (unpublished).

${ }^{8}$ This is really a generalization of the $L_{n}$ problem for $f^{\prime}(z)$.
} 
theorems reduce to theorems of closure for sets of analytic functions. If every function analytic inside and on the contour $C$ can be expanded in a uniformly convergent series of the functions $e^{a_{n} w} \lambda_{n}(w)$, then in particular the function $e^{z w}$ can be so expanded,

$$
e^{z w}=\sum_{n=0}^{\infty} c_{n}(z) e^{a_{n} w} \lambda_{n}(w)
$$

Substituting this expansion into (3) and integrating term by term, we obtain, if $L_{n}\left[f\left(a_{n}\right)\right]=0$,

$$
f(z)=\sum_{n=0}^{\infty} c_{n}(z) \int_{C} e^{a_{n} w} \lambda_{n}(w) \phi(w) d w=\sum_{n=0}^{\infty} c_{n}(z) L_{n}\left[f\left(a_{n}\right)\right]=0 .
$$

Similarly, the generalized uniqueness problem involving univalence leads to the closure problem for the set $\lambda_{n}(w)\left(e^{a_{n} w}-e^{b_{n} w}\right)$, where $a_{n}$ and $b_{n}$ are arbitrary (unequal) points in the circles $C_{n}$.

4. Uniqueness theorems. The most elementary uniqueness theorem is that associated with $L_{n}=L^{n}, L[f(z)]=f^{\prime}(z),\left\{a_{n}\right\}=\{0\}$. Here the theorem states that a function of exponential type-or, of course, any analytic function - which vanishes with all its derivatives at a given point, vanishes identically. An immediate generalization is Whittaker's problem: if $f^{(n)}\left(a_{n}\right)=0,\left|a_{n}\right| \leqq 1$, what is the largest number $A$ such that if $f(z)$ is of type less than $A$ it must vanish identically $[32$, p. 45$]$ ? Since the functions $z^{n} e^{a_{n} z}$ are known [5, pp. 481-482; 15] to form a closed set in $|z|<\log 2$, the general argument just outlined shows that ${ }^{9} A \geqq \log 2$. Pólya has recently shown ${ }^{10}$ that $\log 2$ $<A<0.77<\pi / 4=0.785+.{ }^{11}$ If the $a_{n}$ are further restricted to be real, the corresponding constant $A$ is actually $\pi / 4$ [28]. If we make the restriction $a_{2 n+1}=0,\left|a_{2 n}\right| \leqq 1$, then ${ }^{12}$ the corresponding $A$ exceeds 1.31 .

The univalence theorem corresponding to Whittaker's problem is that one at least of the derivatives of $f(z)$ must be univalent in the unit circle (an infinite number, if $f(z)$ is not a polynomial), provided that $f(z)$ is of type less than $A$, where $A \geqq \log 2[6,18]$; the best possible $A$ is not known.

If $L_{n}[f(z)]=f^{(n)}(z)+b_{n} f(0)$, where the $b_{n}$ are sufficiently small num-

๑ First proved in another way by Takenaka [29].

${ }^{10}$ Unpublished result.

11 Thus disproving the conjecture that $A=\pi / 4$. The example showing that $A<\pi / 4$ is $f(\pi z / 4)$, where $f(z)=e^{z}+\omega^{-1} e^{\omega z}+e^{\omega^{2} z}$ and $\omega^{2}+\omega+1=0$.

12 The result $A \geqq 0.78$ is given in [5, p. 486]; the result stated here can be obtained by modifying the argument on p. 482 . 
bers, we obtain a theorem $\left[5\right.$, p. 485] stating that the numbers $f^{(n)}\left(a_{n}\right)$ cannot all be very small unless $f(z) \equiv 0$. If $L_{2 n}[f(z)]=L_{2 n+1}[f(z)]$ $=f^{(2 n)}(z), a_{2 n}=0, a_{2 n+1}=1$, we have the uniqueness theorem for the so-called two-point boundary problem: if every derivative of even order of a function of exponential type less than or equal to $c$ vanishes both at 0 and at 1 , then $f(z)$ is a sine polynomial of order at most $c / \pi$; if $c<\pi, f(z) \equiv 0 .{ }^{13}$ A similar uniqueness theorem holds for more general sequences $\left\{a_{2 n}\right\},\left\{a_{2 n+1}\right\}$, near 0 and 1 , respectively; the corresponding univalence result also is true.

5. Further uniqueness theorems. When the function $\lambda(w)$ of $(7)$ is univalent inside the contour $C$, we can establish the closure of the functions $\lambda^{n}(w)$ by making a conformal map which carries them into the functions $z^{n}[14]$. In this way it is found, for example, that the operators $L_{n}\left[f\left(a_{n}\right)\right]=\Delta^{n} f(0)$ have a uniqueness theorem for functions of type less than $\log 2$; from this result it can be shown that a function of type less than $\log 2$, taking integral values at the points $n=0,1,2, \cdots$, is necessarily a polynomial. ${ }^{14}$ More generally, the operators $L_{n}\left[f\left(a_{n}\right)\right]=\Delta^{n} f\left(a_{n}\right)$ can be studied; for example, the critical type for the operators $\Delta^{n} f\left(a_{n}\right),\left|a_{n}\right| \leqq 1$, is at least $\log 3 / 2$.

If $\lambda(w)$ is not only univalent, but also has no zeros inside the contour $C$, the sequence of operators $L^{n}$ can be imbedded in the family of operators $L^{t}$ corresponding to the functions $[\lambda(w)]^{t}$, and uniqueness theorems obtained corresponding to various sequences $L^{\lambda_{n}}$. A great deal of attention has been paid to the operator $L[f(z)]=f(z+1)$, corresponding to the function $\lambda(w)=e^{w}$; results for this operator naturally can be transformed by conformal mapping so as to apply to other operators. With this operator the uniqueness problem can be formulated more simply as the problem of finding conditions under which $f\left(\lambda_{n}\right)=0$ implies $f(z) \equiv 0$.

The oldest result of this kind is Carlson's theorem: If $f(z)$ is of exponential type $c, c<\pi,^{15}$ then $f(n)=0(n=0,1,2, \cdots)$ implies that $f(z) \equiv 0 .{ }^{16}$ A more delicate result, applying only to entire functions, states that if

$$
|f(z)|<\epsilon(|z|) e^{\pi|z|}(1+|y|)^{-2 \delta}, \quad 0<\delta<1 / 2, \quad \lim _{r \rightarrow \infty} \epsilon(r)=0,
$$

13 [27]. Another proof in [7]. For generalizations, see [14].

${ }^{14}$ Pólya and Hardy; see [32, p. 55].

${ }^{15} \mathrm{Or}$, more generally, of (some) exponential type in a right half-plane and of type less than $\pi$ along its boundary.

${ }^{16}$ See, for example, [30, p. 186]. A related result of Estermann (Dienes [13, p. 259]) is that if $f(z)$ is of type less than 1 , and $f\left(z_{n}\right)=0,\left|z_{n}\right|=n(n=0,1,2, \cdots)$, then $f(z) \equiv 0$. 
and $f\left(\lambda_{n}\right)=0(n=0,1,2, \cdots)$, where $\left|\lambda_{n}-n\right|<\delta$, then $f(z) \equiv 0 .{ }^{17}$ More general sequences $\left\{\lambda_{n}\right\}$ are allowable if we go back to the stronger requirement that $f(z)$ is of type $c, c<\pi$. For example, if $f(z)$ is of type less than $\pi$ in the half-plane $x \geqq 0$, and if $f\left(\lambda_{n}\right)=0$ where the real numbers $\lambda_{n}$ satisfy $\lim _{n \rightarrow \infty} n / \lambda_{n}=D \geqq 1$, and $\left|\lambda_{n}-\lambda_{m}\right|$ $\geqq|n-m| \delta, \delta>0$, then $f(z) \equiv 0 .{ }^{18}$ Much more general sequences $\left\{\lambda_{n}\right\}$ can be used if the growth of $f(z)$ is restricted by a condition stating roughly that $f(z)$ is of order less than one along the real axis. More precisely, ${ }^{19}$ if $f(z)$ is an entire function of exponential type $c$, such that

$$
\lim _{R \rightarrow \infty} \int_{1}^{R}\left(1 / x^{2}\right) \log |f(x) f(-x)| d x
$$

exists and is finite, and

$$
\limsup _{x \rightarrow \infty}(1 / x) \log |f( \pm x)| \leqq 0,
$$

then the zeros of $f(z)$ in $x \geqq 0$ and in $x \leqq 0$ have a density $B$ at most equal to $c / \pi$. Consequently we cannot have $f\left(\lambda_{n}\right)=0$ for a sequence $\left\{\lambda_{n}\right\}$ whose density exceeds $c / \pi$.

Even more generally, the hypothesis that $f\left(\lambda_{n}\right)=0$ can be replaced by one stating that the numbers $f\left(\lambda_{n}\right)$ are very small $;^{20}$ for example, that $f\left(\lambda_{n}\right)=O\left(e^{-\delta \lambda_{n}}\right), \delta>0$.

Another interesting result is that if (8) is satisfied with $\delta=1$, then $f(z) \equiv 0$ if $f(z)$ vanishes at least once in every interval $(n, n+1)[31$, p. 213]. This suggests that if we let the zeros coalesce in pairs, so that $f(z)$ and $f^{\prime}(z)$ both vanish at $z=2 n$, then $f(z) \equiv 0$ if $f(z)$ is of type less than $\pi$. More generally, we can consider the case where we have two operators $L$ and $M, L[f(2 n)]=M[f(2 n)]=0$, and the functions $\lambda$ and $\mu$ associated with $L$ and $M$ satisfy suitable conditions [4].

6. Growth theorems. Let $L_{t}$ be a one-parameter family of operators, for example the powers of an operator $L$ whose $\lambda(w)$ has no zeros. A uniqueness theorem states that, given sequences $\left\{a_{n}\right\}$ and $\left\{\lambda_{n}\right\}$, any function $f(z)$ of sufficiently small type, such that $L_{\lambda_{n}}\left[f\left(a_{n}\right)\right]=0$, vanishes identically. If $f(z)$ is identically zero, then of course so are $L_{t}[f(z)]$ and $M_{t}[f(z)]$ for every $t$, where $M_{t}$ is another family of operators. We now suppose, not that the elements of the sequence $\left\{L_{\lambda_{n}}\left[f\left(a_{n}\right)\right]\right\}$ are zero, but their growth is restricted in some way;

17 [3, p. 158]. The case $\delta=0$ is due to Pólya [24] and Valiron [31, p. 204].

18 This is an easy deduction from Carleman's theorem (for Carleman's theorem see, for example $[30$, p. 130]).

${ }^{19}$ See $[17$, especially pp. 13,25$]$.

${ }^{20}$ Levinson [17, p. 19]; Levinson gives a number of much more general results. 
and we ask what can be said about the growth of a sequence $\left\{M_{\mu_{n}}\left[f\left(b_{n}\right)\right]\right\}$. The case most extensively studied has been that in which $a_{n}=b_{n}=0$; in this case, it is more convenient to think of the problem as that of determining the growth properties of $\left\{M^{*}\left[f\left(\mu_{n}\right)\right]\right\}$ from those of $\left\{L^{*}\left[f\left(\lambda_{n}\right)\right]\right\}$, where $L^{*}[f(z)]=L_{z}[f(0)]$ and $M^{*}$ is defined similarly.

It is not always desirable to restrict $\left\{\lambda_{n}\right\}$ and $\left\{\mu_{n}\right\}$ to be countable sequences. If we replace both sequences by the set of all real numbers $x$, our original uniqueness problem becomes trivial; but we have the following non-trivial growth problem: given that $L[f(x)]$ is bounded for real $x$, what bound can be assigned to $M[f(x)]$, where $L$ and $M$ are given operators? The oldest case of the problem is S. Bernstein's problem of the bound for the derivative of a function, given a bound for the function; here $L$ is the identity operator and $M[f(z)]=f^{\prime}(z)$. Here we have the result that $\left|f^{\prime}(x)\right| \leqq c K$ if $|f(x)| \leqq K(-\infty<x<\infty) .{ }^{21}$ More generally, if $M$ is an operator with associated function $\mu(w)$ analytic in $|w|<c$, then for every $f(z)$ of type $c^{\prime}<c$ there is a number $A\left(c^{\prime}\right)$, independent of $f$, such that ${ }^{22}$

$$
|M[f(x)]| \leqq A\left(c^{\prime}\right) \sup _{-\infty<x<\infty}|f(x)| .
$$

Under suitable restrictions on $\mu(w)$ on the imaginary axis, it can be shown by use of the representation (5) that $A\left(c^{\prime}\right)$ may be replaced by a number $A(c)$ depending only on $c$. Still more generally we can obtain results of the form

$$
|M[f(x)]| \leqq A\left(c^{\prime}\right) \sup _{-\infty<x<\infty}|L[f(x)]| .
$$

Such a result is no more general than (9) if $L$ has an inverse, that is, if $\lambda(w)$ has no zeros; but may hold even when $\lambda(w)$ does have zeros if the zeros are off the imaginary axis.

7. Growth theorems involving sequences. The theorems to be discussed in this section deduce the growth properties of $M[f(x)]$ from those of $L\left[f\left(\lambda_{n}\right)\right]$. The necessary restrictions on the sequence $\left\{\lambda_{n}\right\}$ vary with the expression taken as measure of the rate of growth. We consider first functions bounded on the real axis. If $f(z)$ is of type $c$, $c<\pi$, and $|f(n)| \leqq K(n=0, \pm 1, \cdots)$, then ${ }^{23}|f(x)| \leqq A(c) K$; the best possible $A(c)$ is $^{24} O(-1 / \log (\pi-c)$ ) as $c \rightarrow \pi$. This result (apart from

${ }^{21}$ See [26, vol. 2, p. 35, section IV, problem 201].

${ }^{22}$ Civin [12], for a restricted class of functions of exponential type. The results can be extended to the general case by the method used in [3, pp. 150-152].

${ }^{23}$ Cartwright [11]. Other proofs by Pfluger [22], Macintyre [20], Boas [3].

${ }^{24}$ Boas and Schaeffer [8]. 
the evaluation of $A(k))$ extends to functions of exponential type in a half-plane, ${ }^{25}$ and in that form carries over directly to other rates of growth, for example, $f(x)=O\left(x^{p}\right)(p>0)$ or $f(x)=O\left(e^{A x}\right)$. However, results involving an exponential rate of growth for $f(z)$ on the real axis extend to more general sequences of points than do those involving merely boundedness for $f(x)$. For boundedness, the most general result so far obtained is that if $\left|\lambda_{n}-n\right|<A$ and $\left|\lambda_{m}-\lambda_{n}\right|$ $\geqq \delta(m \neq n ; m, n=0,1,2, \cdots)$, then $f(z)$, of type less than $\pi$ in $x>0$ and bounded at $\left\{\lambda_{n}\right\}$, is bounded on the real axis. ${ }^{26}$ Results of Levinson $[17$, p. 127] show that one cannot go much farther in this direction. On the other hand, results of the type

$$
\limsup _{x \rightarrow \infty} \frac{\log |f(x)|}{x}=\limsup _{n \rightarrow \infty} \frac{\log \left|f\left(\lambda_{n}\right)\right|}{\lambda_{n}}
$$

hold for sequences $\left\{\lambda_{n}\right\}$ satisfying such general conditions as ${ }^{27}$

$$
\left|\lambda_{n}-\lambda_{m}\right| \geqq|n-m| d, \quad d>0, n / \lambda_{n} \rightarrow D,
$$

where the type of $f(z)$ is less than $\pi D$.

In these results quoted so far in this section, the operators $L$ and $M$ have both been the identity operator. It is clear that more general theorems, at least for the case of boundedness, can be deduced indirectly from the results of $\S 6$. For, from the boundedness of $f\left(\lambda_{n}\right)$ we can infer first that of $f(x)$ and then that of $L[f(x)]$. In this way we can show, for example, that if $|f(n)| \leqq K$ and $f$ is of type $c^{\prime}<\pi$, then $\left|f^{\prime}(x)\right| \leqq c^{\prime} A\left(c^{\prime}\right) K$, where $A\left(c^{\prime}\right)$ is the same as in (9). However, we may in some cases obtain sharper results by proceeding directly; thus Macintyre has pointed out $[20$, p. 6] that $|f(n)| \leqq K$ implies

$$
\left|f^{\prime \prime}(x)+\pi^{2} f(x)\right| \leqq A K,
$$

where $A$ is a constant, even when $f(z)$ is of type $\pi$. The reason for the difference between the operators $L_{1}[f]=f^{\prime}$ and $L_{2}[f]=f^{\prime \prime}+\pi^{2} f$ is clearly shown by the difference between the corresponding functions $\lambda_{1}(w)=w$ and $\lambda_{2}(w)=w^{2}+\pi^{2}$. The second vanishes at $w= \pm i \pi$; it is generally true that an $L$ whose $\lambda$ has this property has the stronger boundedness property typified by (10).

${ }^{25}$ Cartwright [11], Macintyre [20].

${ }^{26}$ Duffin and Schaeffer (unpublished). I am indebted to these authors for permission to include their result in this report.

For a theorem in which the boundedness of $f(z)$ on two sequences implies its boundedness on two lines (and hence that it is a constant) see Levinson [17, p. 122].

${ }^{27}$ For this, and much more general results, see Levinson [17, pp. $100 \mathrm{ff}$.]. Other theorems of this character are given by Junnila [16]. 
8. Integrability. Bernstein's theorem on derivatives states in effect that a function of exponential type, if bounded on a line, cannot oscillate too rapidly on the line. Another illustration of this fact is that if $f(z)$ is of type less than $\pi$ and is bounded on the average near the integers, it is bounded on the real axis; more precisely, if

$$
\sup _{-\infty<n<\infty} \int_{n-}^{n+\epsilon} \psi(|f(t)|) d t<\infty,
$$

where $\epsilon \geqq 0, \delta \geqq 0, \epsilon+\delta>0$, and $\psi(t)$ is non-decreasing and unbounded, then $f(x)$ is bounded [6, p. 163]. In particular, $f(x)$ is bounded (and hence approaches zero) on the real axis if it belongs to $L^{p}$ for some positive $p[23$, p. 124].

A result of similar character is that

$$
\sum_{-\infty}^{\infty}\left|f\left(x_{n}\right)\right|^{p}<\infty, \quad p>0,
$$

with

$$
\left|x_{n}-n\right| \leqq L<\left(2 \pi^{2}\right)^{-1}
$$

implies

$$
\int_{-\infty}^{\infty}|f(x)|^{p} d x<\infty
$$

if ${ }^{28} f(z)$ is of type less than $\pi$; conversely, if $f(z)$ is of any finite type and if the number of $x_{n}$ 's in any interval of unit length is bounded, then (12) implies (11). ${ }^{29}$

Still another indication of the restrictions imposed on the oscillation of $f(x)$ by its integrability properties is that if $f(x)$ belongs to $L^{p}(p>0)$, so does $f^{\prime}(x)[23$, p. 127]. This can be considered as a generalization of Bernstein's theorem mentioned in $\$ 6$; Bernstein's theorem is the limiting case $p \rightarrow \infty$.

\section{REFERENCES}

1. R. P. Boas, Jr., Representations for entire functions of exponential type, Annals of Mathematics, (2), vol. 39 (1938), pp. 269-286; vol. 40 (1939), p. 948.

2. - Remarks on a theorem of B. Lewitan, Matematicheskii Sbornik (Recueil Mathématique), (n.s.), vol. 5 (1939), pp. 185-187.

3. - Entire functions bounded on a line, Duke Mathematical Journal, vol. 6 (1940), pp. 148-169.

4. - - Some uniqueness theorems for entire functions, American Journal of Mathematics, vol. 62 (1940), pp. 319-324.

28 [3, p. 165]; [23, p. 129, with $\left.\lambda_{n}=n\right]$.

$29[3$, p. 153]; a similar result in $[23$, p. 126]. 
5. - Expansions of analytic functions, Transactions of this Society, vol. 48 (1940), pp. 467-487.

6. - Univalent derivatives of entire functions, Duke Mathematical Journal, vol. 6 (1940), pp. 719-721.

7. - A note on functions of exponential type, this Bulletin, vol. 47 (1941), pp. $750-754$.

8. R. P. Boas, Jr., and A. C. Schaeffer, A theorem of Cartwright, Duke Mathematical Journal (to appear).

9. S. Bochner, Vorlesungen über Fouriersche Integrale, Leipzig, 1932.

10. R. D. Carmichael, Functions of exponential type, this Bulletin, vol. 40 (1934), pp. 241-261.

11. M. L. Cartwright, On certain integral functions of order one, Quarterly Journal of Mathematics, Oxford, vol. 7 (1936), pp. 46-55.

12. P. Civin, Inequalities for trigonometric integrals, Duke Mathematical Journal, vol. 8 (1941), pp. 656-665.

13. P. Dienes, The Taylor Series. An Introduction to the Theory of Functions of a Complex Variable, Oxford, 1931.

14. A. Gelfond, Interpolation et unicité des fonctions entières, Matematicheskii Sbornik (Recueil Mathématique), (n.s.), vol. 4 (1938), pp. 115-147.

15. I. I. Ibragimoff (I. Ibraguimoff), Sur quelques systèmes complets de fonctions analytiques (in Russian), Izvestiya Akademii Nauk SSSR, Seriya Matematicheskaya (Bulletin de l'Académie des Sciences de l'URSS, Série Mathématique), 1939, pp. 553-567; French summary, pp. 567-568.

16. A. Junnila, Über das Anwachsen einer analytischen Funktion in einer gegebenen Punktfolge, Suomalaisen Tiedeakatemian Toimituksia (Annales Academiae Scientiarum Fennicae), (A), vol. 48, no. 2 (1936).

17. N. Levinson, Gap and Density Theorems, New York, American Mathematical Society Colloquium Publications, vol. 26, 1940.

18. - A theorem of Boas, Duke Mathematical Journal, vol. 8 (1941), pp. 181-182.

19. B. Lewitan, Über eine Verallgemeinerung der Ungleichungen von S. Bernstein und H. Bohr, Comptes Rendus (Doklady) de l'Académié des Sciences de l'URSS, vol. 15 (1937), pp. 169-172.

20. A. J. Macintyre, Laplace's transformation and integral functions, Proceedings of the London Mathematical Society, (2), vol. 45 (1938-1939), pp. 1-20.

21. R. E. A. C. Paley and N. Wiener, Fourier Transforms in the Complex Domain, New York, American Mathematical Society Colloquium Publications, vol. 19, 1934.

22. A. Pfluger, On analytic functions bounded at the lattice points, Proceedings of the London Mathematical Society, (2), vol. 42 (1936-1937), pp. 305-315.

23. M. Plancherel and G. Pólya, Fonctions entières et intégrales de Fourier multiples Commentarii Mathematici Helvetici, vol. 9 (1936-1937), pp. 224-248; vol. 10 (19371938), pp. 110-163.

24. G. Pólya, Prolongement analytique, L'Enseignement Mathématique, vol. 22 (1922), pp. 298-299.

25. - Untersuchungen über Lücken und Singuläritaten von Potenzreihen, Mathematische Zeitschrift, vol. 29 (1929), pp. 629-640.

26. G. Pólya and G. Szegö, A ufgaben und Lehrsätze aus der Analysis, Berlin, 1925.

27. I. J. Schoenberg, On certain two-point expansions of integral functions of exponential type, this Bulletin, vol. 42 (1936), pp. 284-288. 
28. - On the zeros of successive derivatives of integral functions, Transactions of this Society, vol. 40 (1936), pp. 12-23.

29. S. Takenaka, On the expansion of integral transcendental functions in generalized Taylor's series, Nippon Sûgaku-Buturigakkwai Kizi (Proceedings of the PhysicoMathematical Society of Japan), (3), vol. 14 (1932), pp. 529-542.

30. E. C. Titchmarsh, The Theory of Functions, Oxford, 1932.

31. G. Valiron, Sur la formule d'interpolation de Lagrange, Bulletin des Sciences Mathématiques, (2), vol. 49 (1925), pp. 181-192, 203-224.

32. J. M. Whittaker, Interpolatory Function Theory, Cambridge, 1935.

DUKe UNIVERsity 